\title{
Féminisation dans les textes administratifs écrits en français : le cas de l'Algérie
}

\author{
Feminization of administrative texts: case of Algeria
}

Martin Pleško
Université Palacký à Olomouc, République tchèque

Jan Holeš

Université d'Ostrava, République tchèque

\begin{abstract}
Résumé : L'article traite de la féminisation des textes administratifs écrits en français en Algérie sur la base d'une analyse des formulaires officiels délivrés par diverses autorités publiques algériennes. Après une introduction générale portant sur la situation linguistique et la féminisation linguistique en Algérie, les auteurs présentent ensuite la méthodologie utilisée. Les résultats sont résumés, commentés et quantifiés en tenant compte des procédés appliqués (ou non) visant à rendre les femmes visibles dans ce type de documents.
\end{abstract}

Mots-clés : Algérie, féminisation, formulaires, langue administrative, français, religion.

Abstract: The article deals with the feminization of French administrative texts in Algeria on the basis of an analysis of official forms issued by various Algerian public authorities. A general introduction dealing with the linguistic situation and the linguistic feminization in Algeria is followed by the explication of the used methodology. Results are resumed, commented and quantified according to various processes applied (or not applied) to make the women visible in this type of texts.

Keywords: Algeria, feminization, forms, administrative language, French, religion.

\section{Introduction}

Cet article s'inscrit dans le domaine de la sociolinguistique et a pour but d'examiner les états des lieux en matière de féminisation linguistique du français utilisé en Algérie. À notre connaissance, il n'y a pas, à l'heure actuelle, d'études traitant de la visibilité (ou de l'invisibilité) linguistique des Algériennes d'un point de vue purement linguistique ou sociolinguistique. Comme l'étude de la féminisation linguistique est un objet d'intérêt à travers le monde francophone en Europe et en Amérique, nous pensons qu'il est aussi important de la décrire dans le contexte africain.

Notre recherche se limite au discours administratif, dont les formulaires représentent un exemple caractéristique, et à la féminisation lexicale, cette dernière ayant pour but la formation des unités lexicales féminines. II s'agit donc d'une tâche d'ordre lexicologique (reposant principalement sur le procédé de dérivation) et de nombreux guides ont été publiés pour présenter les règles basiques de formation des formes féminines. 
L'Algérie jouit de son indépendance depuis presque soixante ans et la nécessité s'impose de dresser le bilan: Que sont devenues les femmes algériennes au niveau de la langue française? Devrions-nous parler d'une égalité ou d'une inégalité linguistique entre femmes et hommes d'Algérie ? À quel point sont-elles (in)visibles dans la langue française qui est en usage dans leur patrie ? Par quels moyens linguistiques sont-elles rendues (in)visibles dans le corpus des formulaires institutionnels en langue française utilisés en Algérie?

\section{La situation linguistique en Algérie}

La situation linguistique et la position du français au Maghreb et en Algérie ont été maintes fois décrites et analysées (voir par ex. Kadlec, 2012 : 13-51 ; Kadlec - Holeš, 2012 : 77-90 ; Lanly, 1962 ; Laroussi, 1997 ; Queffélec - Benzakour - Cherrad-Benchefra, 1995). L'ouvrage de Queffélec, Derradji, Debov, Smaali-Dekdouk et CherradBenchefra (2002) présente une analyse incontournable menée à travers la description de la situation du français en Algérie. Ce livre contient un inventaire de 1500 algérianismes, comprenant des emprunts à l'arabe, surtout dans le domaine de la religion, de l'administration, de la nourriture, de la tenue vestimentaire et des objets de la réalité quotidienne, des néologismes de forme, des néologismes sémantiques et, entre autres, des spécificités de la féminisation de certains noms (agente: "femme de service», agente de police: "femme agent de police»). Ajoutons encore que la Base de données lexicographiques panfrancophone, disponible en ligne, contient aussi des particularités du français algérien.

Comme le rappelle Leclerc (2015), l'Algérie constituerait la seconde communauté francophone du monde. En fait, l'Algérie serait, après la France, le premier pays francophone au monde, avec plus de 14 millions d'individus de 16 ans et plus qui pratiquent le français, soit $60 \%$ de la population. Or le même auteur ajoute que les recensements sur une base linguistique, ethnique ou religieuse sont interdits en Algérie afin de préserver l'unité nationale et qu'il est donc malaisé de connaître de façon scientifique le nombre exact de locuteurs des langues présentes sur son territoire. Soulignons que malgré les effectifs élevés de locuteurs, le français ne bénéficie pas d'un statut officiel en Algérie.

Une forte tradition de la langue française et un nombre important de locuteurs et locutrices du français justifient la nécessité de s'intéresser à toutes les particularités (socio)linguistiques qui pourraient éventuellement relever de l'usage du français en Algérie.

\section{Pour comprendre la féminisation linguistique: les Algériennes et la langue française}

La féminisation linguistique est, entre autres, une revendication fondamentale de tout être humain, pas seulement de ceux du sexe féminin. Au-delà de la visibilité linguistique des femmes, elle vise également à atteindre l'égalité linguistique entre femmes et hommes. Sur le plan social, elle veut (ou devrait) témoigner de la place prise par les femmes dans les sociétés contemporaines. En effet, c'est un objet d'étude qui jouit d'une assez longue tradition en Belgique, en France, au Québec, en Suisse et ailleurs (Elmiger, 2008 ; Houdebine-Gravaud, 1998 ; Labrosse, 1996, 2002, 2005 ; Larivière, 2000-a, b ; Pleško, 2012, 2015). Au Québec, la féminisation linguistique est déjà bien implantée dans le quotidien. La France en revanche garde toujours une position plutôt conservatrice. 
La féminisation en français dépasse les seules considérations linguistiques et touche à la représentation identitaire et sociale. II s'agit donc d'une question sociolinguistique recouvrant des enjeux sociétaux (point de vue socio-économique, philosophique et/ou politique) et individuels (point de vue psychologique, psycholinguistique et souvent sexuel). La question est donc de savoir: Pourquoi étudier la féminisation linguistique dans les territoires francophones en Afrique? Parmi les différents facteurs explicatifs de ce phénomène, on peut citer tout d'abord l'absence d'influence de l'Académie française sur les règles du français en Algérie et la différence entre les caractéristiques culturelles et linguistiques de la nation algérienne et celles des territoires francophones d'Europe et d'Amérique du Nord. II faut mentionner par ailleurs l'impact des langues locales sur la féminisation du français algérien'. Enfin, il ne faut pas omettre l'impact de l'Islam sur la langue française puisque l'Algérie est majoritairement musulmane. Le 27 février 2005, le président de la République algérienne Abdelaziz Bouteflika a signé l'Ordonnance ${ }^{2} n^{\circ}$ 05-02 du 27 février 2005 modifiant et complétant la loi n 84- 11 du 9 juin 1984 portant (sic !) code de la famille publiée dans le Journal officiel de la République algérienne (JORA) $(2005,17)$. Promulguée en 1984, cette loi légalise un statut différencié entre hommes et femmes, et place ces dernières en position de subordination. Elle confère des pouvoirs au mari et au père. Les faits sociaux relatifs à cette inégalité des sexes à quasiment tous les niveaux de la vie d'un être humain présentent un champ extrêmement vaste. Pour les femmes, il est difficile d'affronter des pratiques inégalitaires imprégnant toute la société (Lalami, 2012 : 15-16).

Ces pratiques pourraient éventuellement causer l'invisibilité des femmes algériennes non seulement dans le fonctionnement de la société, mais aussi dans la langue française utilisée en Algérie.

\section{Méthode}

Bien que les formulaires soient très présents dans notre vie quotidienne, nous n'en trouvons que relativement peu de mentions dans les ouvrages de référence. Les formulaires concernent une grande partie de la population, même si peu de personnes participent à leur élaboration. Lamothe et al. (1992) affirment que ce type de documents écrits comporte une syntaxe très simple (pourtant, les formulaires contiennent quelques instructions, consignes ou explications nécessaires pour les remplir). II n'y a donc que peu de termes à féminiser, surtout lorsque nous optons, lors de la rédaction, pour des solutions alternatives aux doublets et pour une mise en page adéquate.

Nous avons réuni un corpus de dix formulaires algériens. Nos premières observations nous ont convaincu de ne pas en analyser un plus grand nombre parce que ce type de texte est assez répétitif. Pour constituer ce corpus, nous avons veillé aux critères suivants :

\footnotetext{
1 Nous nous servons de la dénomination français algérien juste pour le besoin du présent article.

2 Selon cette ordonnance, la femme majeure ne peut pas conclure son mariage sans wali (tuteur matrimonial), et ne peut obtenir le divorce que sous des conditions limitées; la polygamie est maintenue. "Art. 11 La femme majeure conclut son contrat de mariage en présence de son "wali" qui est son père ou un proche parent ou toute autre personne de son choix. Sans préjudice des dispositions de l'article 7 de la présente loi, le mariage de la mineure est contracté par le biais de son "wali", qui est le père, puis la femme majeure conclut son contrat de mariage en présence de son "wali" des proches parents. ॥ (Lalami, 2012 :16).
} 
1) Les formulaires sont téléchargeables depuis Internet.

2) Le réseau Internet doit être destiné à un public francophone et le texte doit être rédigé en français. Les cas bilingues franco-arabe ont été acceptés pour des raisons évidentes.

3) Les formulaires concernent des individus, il ne s'agit pas de personnes morales.

4) Les formulaires sont produits par les autorités algériennes. Les formulaires issus des institutions francophones opérant en Algérie ont été exclus.

Pour ce qui est de la constitution du corpus, notre choix comporte aussi des faiblesses. D'abord, le risque de subjectivité est considérable. Afin de le réduire autant que possible, nous n'avons lu aucun texte dans sa totalité en créant le corpus, pour que son contenu ne nous influence pas à priori. Nous nous sommes limité à la lecture du titre pour être sûr qu'il s'agisse d'un formulaire institutionnel. Cependant, même le titre a parfois une valeur suggestive, car certains procédés de féminisation linguistique s'y manifestent déjà: Demande de carte nationale d'identité (à remplir par le demandeur). Se pose donc la question de savoir si ce formulaire n'est destiné qu'aux hommes algériens ou si l'unité lexicale le demandeur fonctionne en tant que masculin générique. II est impossible de répondre à ce type de question avant de faire une analyse détaillée des formulaires.

\section{Résultats}

Dans les textes choisis, nous avons observé les moyens linguistiques employés pour que les femmes soient présentes et visibles dans la langue. Les résultats sont résumés dans le tableau récapitulatif présenté ci-dessous et accompagné d'exemples, de commentaires et de cas particuliers ou difficiles à classer. Nous avons emprunté cette méthodologie à Pleško (2015 : 104-129) qui présente les procédés linguistiques de féminisation, de mixation et de dégenrisation ${ }^{3}$ propres à la langue française.

\begin{tabular}{|l|l||c|}
\hline \multicolumn{2}{|c|}{ Procédé } & Nombre \\
\hline \multirow{2}{*}{ Épicènes / bivalents } & Épicènes dits propres & 3 \\
\cline { 2 - 3 } & Épicènes employés au masculin & 3 \\
\hline Doublets & Doublets abrégés & 15 \\
\cline { 2 - 3 } & Doublets intégraux & 0 \\
\hline Couples & Génériques masculins & 10 \\
\hline Apposition lexicale (femme ou homme) & 38 \\
\hline Génériques $^{4}$ & & 0 \\
\hline
\end{tabular}

\footnotetext{
3 Féminiser signifie introduire des formes féminines à côté de celles masculines. Mixer signifie alterner des genres linguistiques dans un texte (par ex. : règles d'agencement, alternance des genres, règle de proximité). Dégenriser signifie effacer la marque du genre linguistique.

4 Dans cette perspective, nous pourrions recourir aux travaux de Jespersen (1971 : 319-324) qui postule très explicitement la nécessité d'avoir des termes génériques ne signalant pas le sexe pour parler des êtres vivants, et par conséquent des humains : "Lorsqu'on parle d'êtres vivants, il est souvent souhaitable, et même nécessaire, de disposer de mots qui ne précisent pas le sexe et qui peuvent s'appliquer aussi bien à des êtres mâles qu'à des êtres femelles $॥$.
} 


\begin{tabular}{|c|c|}
\hline Génériques féminins & 0 \\
\hline Unisexués & 0 \\
\hline Bisexués & 1 \\
\hline Note & 0 \\
\hline Abréviation (H/F ou autre) & 1 \\
\hline Dégenrisation & 0 \\
\hline Total & 71 \\
\hline
\end{tabular}

Afin de mieux comprendre les procédés linguistiques productifs présents dans notre corpus, notons que par épicène, nous entendons un nom dont la forme ne varie pas selon le genre. La sous-catégorie des épicènes proposée par Pleško (2015), à savoir les épicènes dits propres ou bivalents, regroupe les noms accompagnés ou non de déterminants et/ou d'adjectifs féminins ou masculins, qui ont donc la qualité d'un féminin et d'un masculin en même temps. Par contre, les épicènes que le déterminant ou les mots accompagnants rendent soit masculins, soit féminins, fonctionnent en fait comme des noms génériques (féminins ou masculins selon le cas). Les doublets peuvent être abrégés (un-e étudiant(e), le/la directeur-trice, etc.) ou intégraux (les étudiants et étudiantes; le directeur/la directrice; etc.). Les couples sont les formes possédant des équivalents masculins/féminins indépendants. Les bisexués sont les formes ne variant pas en genre et qui s'emploient pour les deux sexes. L'abréviation peut être H/F dans les offres d'emploi ou ailleurs, $M$ - $F$ pour déterminer le sexe, etc.

À partir du tableau récapitulatif introduit plus haut montrant les procédés féconds appliqués dans les dix formulaires analysés, nous pouvons constater que les femmes algériennes sont linguistiquement plutôt invisibles dans 41 cas : 3 épicènes employés au masculin +38 génériques. En revanche, il convient de souligner la représentation égale des genres linguistiques féminin et masculin ${ }^{5}$, mais dans 30 cas seulement : 3 épicènes dits propres +15 doublets +10 couples +1 bisexué +1 abréviation .

\begin{tabular}{|l|l|}
\hline Égalité des genres linguistiques & 30 \\
\hline Seulement le genre linguistique masculin & 41 \\
\hline Seulement le genre linguistique féminin & 0 \\
\hline
\end{tabular}

En ce qui concerne la fécondité des procédés linguistiques assurant l'égalité des genres linguistiques, nous constatons que le masculin générique est le procédé le plus fréquent (38). Suivent les doublets (15). D'autres procédés sont également assez productifs : c'est le cas des couples (10) et des épicènes ou des bivalents (6). Peu productifs sont les bisexués (1) et l'abréviation (1).

\footnotetext{
5 Par genre linguistique, nous entendons une propriété de la sphère du groupe nominal reposant sur la règle de l'accord qui s'applique au déterminant, à l'adjectif épithète ou attribut, parfois au participe passé, ainsi qu'au pronom représentant le nom.
} 
Dans les passages suivants, nous donnerons quelques exemples des unités repérées correspondant à tel ou tel procédé linguistique. Pour les épicènes, il y en a trois dits propres : célibataire, des jeunes; et trois épicènes employés au masculin : à l'égard du redevable concerné, le médecin, qui fonctionnent donc en tant que masculins génériques et ne contribuent nullement à la parité linguistique. Les doublets sont toujours et dans tous les formulaires sous forme abrégée et parenthétisée: né(e) le..., le nommé(e), marié(e), veuf(ve), divorcé(e), etc. Les couples les plus fréquents sont : fils de... et fille de..., et : prénom(s) du père... nom et prénom(s) de la mère, ce qui relève de l'identité culturelle et religieuse de l'Algérie. Pour le bisexué et l'abréviation, il s'agit de : personne, et de : $M-F$. Cette dernière abréviation sert à spécifier le sexe de l'individu: $M$ pour masculin/mâle, et $F$ pour féminin/femelle.

Suivent deux cas de figure particuliers. Dans le formulaire intitulé Demande de carte nationale d'identité (à remplir par le demandeur) figurent les deux consignes suivantes pour le remplir correctement: Pour les femmes mariées, inscrire seulement le nom de jeune fille à cette rubrique (rubrique: nom); En cas de pluralité de mariages, le demandeur féminin devra fournir tous renseignements complémentaires (rubrique: nom et prénoms du conjoint [sic !]). Dans le contexte global, ces deux notes peuvent se voir attribuer une connotation péjorative, voire discriminatoire.

Dans un autre formulaire, nous trouvons : Par devant nous, le Chargé des Affaires Consulaires (...). Cela semble un élément limitant l'accès des femmes à ce poste. En général, on soutient le fait que, excepté un formulaire sur neuf, aucun autre n'est rédigé uniquement au masculin générique. Pourtant, il arrive que l'on retrouve des formulations comme : (...) déclarés par le redevable $M . . . .,(.$.$) atteste que M . . .$. etc. Ensuite, l'état civil n'est pas souvent indiqué par une croix dans la case correspondante, mais on l'écrit en lettres à la main. Cela permet d'éviter des doublets dits abrégés et/ou intégraux et de choisir entre une forme masculine/féminine et une forme générique (normalement masculine). Suivant la tradition du monde musulman, la majorité des formulaires contient la rubrique fils(le) de... ; fils/fille de..., fils de... et de..., fils/fille de... et de..., etc. Ces formes sont classées en tant que couples. Le premier exemple pourrait éventuellement donner lieu à une nouvelle sous-catégorie de couples, à savoir les "couples abrégés", même s'il ne s'agit pas de dérivation du féminin à partir du masculin. Cet emploi s'explique par l'économie d'expression tout à fait caractéristique de ce type de texte.

\section{Conclusion}

L'actuelle Constitution de l'Algérie a été adoptée en 1996 et modifiée en 2002, 2008 et en 2016. Son texte original contenait déjà, dans son Article 34, la formulation suivante :

Les institutions ont pour finalité d'assurer l'égalité en droits et devoirs de tous les citoyens et citoyennes en supprimant les obstacles qui entravent l'épanovissement de la personne humaine et empêchent la participation effective de tous, à la vie politique, économique, sociale et culturelle.

Or il est possible de constater que les formulaires d'origine algérienne n'atteignent pas encore la parité linguistique des genres. On a ainsi recensé 30 formes employées à la fois au féminin et au masculin ( $\rightarrow$ parité linguistique des genres) et 41 formes employées uniquement au masculin. 
Les formulaires présentent souvent des inconséquences et, parfois, des contradictions qui ne seront éliminées qu'après l'adoption potentielle d'un texte législatif régissant leur rédaction. Pourtant, le phénomène de féminisation linguistique est présent dans la société algérienne, même si les approches en la matière ne sont pas aussi systématiques et intensives qu' au Canada, en France ou ailleurs.

Enfin, citons Uvírová (2003 : 97-98) :

[l]es femmes sont soumises au statut éternel de la féminité. Aimez, travaillez, écrivez, soyez femmes d'affaires ou de lettres, mais rappelez-vous toujours que l'homme existe et que vous n'êtes pas faites comme lui : votre ordre est libre à condition de dépendre du sien [...].

Selon nous, cet extrait fait plutôt référence à la société européenne et américaine ayant un code de la famille qui ne réduit pas la femme à la dépendance de l'homme. Certes, nous pourrions présupposer une nouvelle perception de la femme algérienne après le changement du code de la famille de 1984 modifié par l'ordonnance mentionnée dans les paragraphes précédents qui semble être, en quelque sorte, en opposition avec la Constitution actuelle.

\section{Corpus (liste des formulaires analysés)}

(Tous les formulaires en ligne ont été consultés le 15 juin 2015)

1) Ministère des Affaires étrangères. Demande d'inscription sur la liste électorale

http://consulatalgeriemontreal.com/v1.0/services_aux_algeriens/formulaires/inscripti on liste electorale.pdf

2)Ministère des Affaires étrangères. Autorisation paternelle (délivrée à des mineurs de nationalité algérienne)

http://www.algerie.uz/autori1.pdf

3) Ambassade d'Algérie, Prague. Procuration

http://www.algerie.cz/images/pdfs/Procuration.pdf

4) Ministère des Affaires étrangères. Demande de visa

http://www.mae.gov.dz/documents/68.aspx

5)Formulaire de demande du passeport et de la carte nationale d'identité

http://www.interieur.gov.dz/PublishingFiles/formulaire_Bio 1.pdf

6) Fiche de renseignement pour la participation au concours sur épreuves pour l'accès au grade de...

http://www.concours-fonctionpublique.gov.dz/images/imprime\%20concours\%20sur\%20epreuves\%20francais.pdf

7)ANSEJ. Formulaire d'inscription

http://www.ansej.org.dz/sites/default/files/formulaire fra.pdf

8) Wilaya de Tipasa. Daira de Hadjout. Certificat médical de bonne santé 
http://www.dairahadjout.com/download/doc/100077.pdf

9) Caution bancaire

http://www.mfdgi.gov.dz/telechargement.php\#

10) Ministère des finances. Direction générale des impôts. Bordereau - avis de versement

http://www.mfdgi.gov.dz/index.php/telechargements

\section{Bibliographie}

Base de données lexicographiques panfrancophone, http://www.bdlp.org (consulté le 28/01/2017).

ELMIGER, D. (2008) : La féminisation de la langue en français et en allemand. Querelle entre spécialistes et réception par le grand public. Paris: Honoré Champion.

HOUDEBINE-GRAVAUD, A.-M. (dir.) (1998) : La féminisation des noms de métiers en français et dans d'autres langues. Paris : L'Harmattan.

JESPERSEN, O. (1971) : La philosophie de la grammaire. Paris : Les Éditions de Minuit.

KADLEC, J. (2012): Francouzština $\vee$ Africe. Olomouc : Univerzita Palackého $\vee$ Olomouci.

KADLEC, J. \& J. HOLEŠ (2012): Jazyková politika frankofonních zemí. Olomouc: Univerzita Palackého v Olomouci.

LABROSSE, C. (1996): Pour une grammaire non sexiste. Québec: Les éditions du remue-ménage.

- (2002) : Pour une langue française non sexiste. Québec : Les Intouchables.

- (2005) : Langage non sexiste, http://http//www.langagenonsexiste.ca/menu.htm (consulté le 18 janvier 2017).

LALAMI, F. (2012) : Les Algériennes contre le code de la famille. Paris: Presses de Sciences Po.

LAMOTHE, J. (dir.) (1992): Guide de féminisation. Montréal: Université du Québec à Montréal, https://instances.uqam.ca/Guides/Pages/GuideFeminisation.aspx (consulté le 17 mars 2017).

LANLY, A. (1962) : Le Français d'Afrique du Nord. Paris : Bordas-PUF.

LARIVIÈRE, L.-L. (2000-a) : Comment en finir avec la féminisation linguistique ou les mots pour LA dire. Paris : Éditions 00h00.

- (2000-b) : Pourquoi en finir avec la féminisation linguistique ou à la recherche des mots perdus. Montréal : Boréal.

LAROUSSI, F. (1997) : Plurilinguisme et identités au Maghreb. Roven: Publications de I'Université de Roven.

LECLERC, J. (2015) : Algérie. L'aménagement linguistique dans le monde. Québec: Université Laval, http://www.tlfa.ulaval.ca/axl (consulté le 23 janvier 2017).

PLEŠKO, M. (2012) : La féminisation linguistique en milieu francophone. Romanica Olomucensia, 24, p. 151-158.

- (2015) : Les femmes, le français et la francophonie. La féminisation linguistique en Belgique, en France, au Québec et en Suisse. Olomouc : Univerzita Palackého $\checkmark$ Olomouci. 
QUEFFÉLEC, A., F. BENZAKOUR \& Y. CHERRAD-BENCHEFRA (1995): Le français au Maghreb. Aix-en-Provence : Publications de l'Université de Provence.

QUEFFÉLEC, A., Y. DERRADJI, V. DEBOV, D. SMAALI-DEKDOUK \& Y. CHERRADBENCHEFRA (2002) : Le français en Algérie. Lexique et dynamique des langues. Bruxelles : Duculot.

UVÍROVÁ, J. (2003) : "Ève extraite de la côte d'Adam? À propos du féminisme surtout grammatical ». Acta Universitatis Palackianae Olomucensia, Facultas Philosophica, Philologica 80 - Romanica Olomucensia, 12, p. 89-100. 